\title{
The gut microbial composition in polycystic ovary syndrome with insulin resistance: findings from a normal-weight population
}

\author{
Fangfang He and Yumei Li
}

\begin{abstract}
Background: Limited studies have reported the relationship between intestinal flora dysbiosis and clinical characteristics in polycystic ovary syndrome (PCOS). However, the structure and characteristics of gut microbiota in PCOS have not been fully elucidated.

Objective: To analyze the composition of the Intestinal flora population in normal-weight women with PCOS and insulin resistance(IR) compared to PCOS alone and healthy women.

Methods: A total of 14 PCOS patients with insulin resistant(PCOS-IR) and 12 PCOS alone (PCOS-NIR), and 10 ageand body mass index-matched healthy control women (HC). BMl: $18.5-23.9 \mathrm{~kg} / \mathrm{m}^{2}$. The bacterial $16 \mathrm{~S}$ rDNA V3-V4 fragment was amplified and sequenced. Then, the sequencing data were analyzed for species annotation, community diversity, and inter-group differences, to explore gut microbial characteristics of the subjects and their correlation with clinical parameters.

Results: No significant difference in diversity was observed between PCOA and sample cluster analysis among the three groups (Beta-diversity) and Alpha-diversity. The relative abundance of Rothia, Ruminococcus, and Enterococcus was significantly higher in the PCOS-IR group than in the other two groups $(P<0.05)$, while that of Prevotella was dramatically decreased $(P<0.05)$. The abundance of Enterococcus was positively correlated with waist circumference, hip circumference, diastolic blood pressure, and insulin resistance index. Meanwhile, Rothia abundance is positively associated with waist circumference and free fatty acids.

Conclusions: The gut microbial composition of PCOS patients with insulin resistance is different from that of PCOS alone and healthy women. The difference is correlated with the clinical characteristics of PCOS, with regards to insulin resistance, abdominal obesity, free fatty acids, and other indicators. PCOS-IR patients have an increased abundance of Enterococcus which potentially the intestinal environment of the host by enriching the metabolic pathways related to insulin resistance, causing the occurrence and development of PCOS.
\end{abstract}

Keywords: Polycystic ovary syndrome, Gut microbiota, Insulin resistance, Enterococcus

\section{Introduction}

Polycystic ovary syndrome (PCOS) is a common endocrine and metabolic disease in women, related to hirsutism, hyperandrogenism, ovulation dysfunction, menstrual

\footnotetext{
* Correspondence: liyumei@csu.edu.cn

Department of Assisted Reproduction, Xiangya Hospital, Central South University, 410008 Changsha, People's Republic of China
}

disorders, and infertility [1]. About $50-70 \%$ of cases of anovulatory infertility in patients are linked to PCOS [2], specifically those accompanied by low ovulation induction rate, low pregnancy rate, and high abortion rate. PCOS is associated with several metabolic disorders, including insulin resistance (IR), obesity, cardiovascular disease, diabetes, and other long-term metabolic syndromes. At

(c) The Author(s). 2021 Open Access This article is licensed under a Creative Commons Attribution 4.0 International License, which permits use, sharing, adaptation, distribution and reproduction in any medium or format, as long as you give appropriate credit to the original author(s) and the source, provide a link to the Creative Commons licence, and indicate if changes were made. The images or other third party material in this article are included in the article's Creative Commons licence, unless indicated otherwise in a credit line to the material. If material is not included in the article's Creative Commons licence and your intended use is not permitted by statutory regulation or exceeds the permitted use, you will need to obtain permission directly from the copyright holder. To view a copy of this licence, visit http://creativecommons.org/licenses/by/4.0/ The Creative Commons Public Domain Dedication waiver (http://creativecommons.org/publicdomain/zero/1.0/) applies to the data made available in this article, unless otherwise stated in a credit line to the data. 
present, the etiology and pathogenesis of PCOS are still unknown, which may involve lifestyle, neuroendocrine, genetic factors, immune and metabolic dysfunction [3]. Insulin resistance is considered to be the main pathological basis of reproductive dysfunction in polycystic ovary syndrome,independent of obesity [4-8]. Notably, insulin resistance and hyperinsulinemia affect testosterone synthesis and secretion, while hyper androgen levels can lead to hirsutism, acne, ovulation disorders, and menstrual disorders. On the other hand, insulin resistance exhibits a long-term and severe effect on metabolism in patients with polycystic ovary syndrome [9].

Intestinal flora,the "second genome" obtained by the human body, co-evolves with the host to promote metabolism and immune response [10]. Evidence indicates that gut microbiome disorders are closely related to the occurrence and development of metabolic diseases, including PCOS $[11,12]$. Also, several studies have attributed the appearance of IR to gut microbiota dysbiosis $[13,14]$. The changes of intestinal flora potentially affect insulin sensitivity by regulating chronic inflammation mediated by lipopolysaccharide, branched chain amino acid, short chain fatty acid (SCFA), and bile acid metabolism, and stimulating the secretion of enterocerebral peptides, resulting in insulin resistance and hyperinsulinemia [15-17]. The structure and characteristics of intestinal microbiota in patients with PCOS have not been comprehensively elucidated [18-22]. Besides, only limited studies have explored the relationship between intestinal flora dysbiosis, clinical characteristics and metabolism in patients with PCOS.

Intestinal flora imbalance causes IR, which is closely linked to the occurrence of PCOS. Herein, excluding the influence of obesity, we conducted a pilot study to examine the correlation between gut microbiota, insulin resistance, and clinical characteristics of PCOS patients. These findings might provide novel insights on the mechanism of occurrence and development of PCOS, thus, accelerating the formulation of new approaches for the prevention and treatment of PCOS.

\section{Materials and methods \\ Participants}

A total of 26 women with polycystic ovary syndrome (PCOS) aged 18-35 years who visited the Department of Assisted Reproduction (Xiangya Hospital, Central South University) between August and December 2019 were recruited. Additionally, we recruited 10 normal women visiting a similar department during the same period for assisted reproduction due to the "male-factor" or "fallopian tube factor" were enrolled as the control group. This study was approved by the Ethics Committee of the Department of Assisted Reproduction (Xiangya Hospital, Central South University)and China Registered Clinical
Trial Ethics Review Committee (Ethical Review No.: CHiECRT1900028223). All participants provided written informed consent. The participants were non-obese women (BMI $18.5-23.9 \mathrm{~kg} / \mathrm{m}^{2}$ ) [23, 24]. PCOS diagnosis was conducted as per the Rotterdam criteria revised during the 2003 Conference; PCOS was diagnosed if two out of the following three features were present: Oligoovulation or amenorrhea; clinical manifestations of androgen and/or biochemical hyperandrogenism (HA); polycystic ovary: ultrasound reports show that the follicles of unilateral or bilateral ovaries with a diameter of $2-9 \mathrm{~mm}$ are larger than 12 , and/or the volume of the ovary $\geq 10 \mathrm{ml}$ [7]. Individuals excluded from one of the following conditions: Cushing syndrome, congenital adrenocortical hyperplasia, androgen-secreting tumors. None of the subjects were treated with hormone drugs, insulin sensitizers, antibiotics, probiotics and prebiotics, traditional Chinese medicines, and immunosuppressants less than three months before the study. Based on the Rotterdam criteria, women in the control group had no history of menstrual disorder, endocrine diseases, or diagnosed PCOS. The HOMA-IR index was calculated as follows: fasting insulin (FINs, mIU/L) fasting plasma glucose (FPGs, $\mathrm{mmol} / \mathrm{L}$ )/22.5. Insulin resistance was defined as fasting insulin $>10 \mathrm{mU} / \mathrm{ml}$, or HOMA-IR > 1.66 , or abnormal insulin release curve (insulin peak more than ten times the basic value; insulin peak delayed to $1 \mathrm{~h}$ after taking sugared water; area under the insulin curve increased; insulin level not returning to the normal fasting level $3 \mathrm{~h}$ after taking sugared water), but with normal fasting blood glucose and glucose tolerance levels [25].

\section{Sampling}

Data regarding anthropometry and metabolic parameters were collected for all participants, including: (1) measurements, including height, weight, waist circumference, hip circumference, and blood pressure, (2) detection of biochemical indicators, such as the sex hormone levels, including progesterone $\left(\mathrm{P}_{4}\right)$, prolactin (PRL), testosterone(T), luteinizing hormone (LH), follicle-stimulating hormone (FSH), oestradiol (E2), sex hormone binding globulin (SHBG), Anti-Mullerian hormone (AMH), and glucose and insulin levels during an oral glucose tolerance test on the third day of the menstrual cycle (In the case of amenorrhea patients, blood samples were collected on any day of the menstrual cycle). Meanwhile, biochemical indices, including triglyceride (TG), total cholesterol (TC), lowdensity lipoprotein (LDL), high-density lipoprotein (HDL), total bile acid, free fatty acid, and inflammatory markers such as C-reactive protein (CRP), interleukin-6 (IL-6), and tumor necrosis factor (TNF- $\alpha$ ) were measured. (3) Feces samples were collected from all patients 
after menstruation, and the samples were immediately frozen and stored at $-80^{\circ} \mathrm{C}$ until analysis.

\section{DNA extraction and PCR amplification}

Following the manufacturer's instructions, microbial DNA was extracted from stool samples using a TIANgen stool DNA kit. The $16 \mathrm{~S}$ rDNA V3 + V4 region of the ribosomal RNA gene was amplified by polymerase chain reaction (PCR). The V3-V4 variable region of the bacterial 16sRNA was amplified via PCR using (5'-CCTA CGGRRBGCASCAGKVRVGAAT-3') and (5'-GGACTA CNVGGGTWTCTAATCC-3') primers under the following conditions: denaturing at $94{ }^{\circ} \mathrm{C}$ for $3 \mathrm{~min}$ followed by 24 cycles of $94{ }^{\circ} \mathrm{C}$ for $5 \mathrm{~s}, 57^{\circ} \mathrm{C}$ for $90 \mathrm{~s}$, and $72{ }^{\circ} \mathrm{C}$ for $10 \mathrm{~s}$, then a final extension at $72{ }^{\circ} \mathrm{C}$ for $5 \mathrm{~min}$. Each PCR reaction mix $(25 \mu \mathrm{L})$ included $2.5 \mu \mathrm{L}$ TransStart Buffer, $2 \mu \mathrm{L}$ dNTPs, $1 \mu \mathrm{L}$ primer $(2 \mu \mathrm{M}), 0.5 \mu \mathrm{L}$ TransStart Taq DNA and 20 ng of DNA template. The PCR products were assessed using $1.5 \%$ agarose gel electrophoresis, quantified by Qubit3.0 Fluorometer (Invitrogen, Carlsbad, CA), then pair-end sequenced on Illumina Miseq PE250 platform (Illumina, San Diego, CA, USA).

\section{Next-generation sequencing}

Filtration of raw tags was performed using QIIME (V1.9.1) to dislodge the noisy sequences [26]. The filtered clean tags were searched against the Gold database to identify chimeric tags, which were then removed using the UCHIME algorithm to obtain Effective Tags. Based on the Effective Tags of each sample, the OUT (Operational Taxonomic Units) were clustered using QIIME software (Version 1.9.1) based on the GreenGene database [26-28]. Alpha diversity was reflected by Chao1, Observed OTUs,Simpson, and Shannon indexes. Beta diversity was presented on principal coordinates analysis (PCoA) charts created using PCoA statistical analysis method with $\mathrm{R}$ language(version 3.3.3). Based on the species abundance table, the $P$-value was obtained by Kruskal-Wallis $\mathrm{H}$ test analysis and then modified by Benjamini and Hochberg False Discovery Rate method to obtain the $Q$ value. The species with significant differences between groups were obtained with $Q$ value $<0.05$ as the threshold [29]. Linear discriminant analysis (LDA) effect size (LEfSe) was used to identify the bacterial taxa and metabolism-associated clinical parameters with significant differences between groups. Logarithmic LDA values $>2.0$ and $P<0.05$ were set as the threshold for differential flora identification.

\section{Statistical analysis of clinical data}

Data were analyzed using SPSS 22.0. Quantitative demographic and clinical data with normal distribution were presented as the mean \pm standard deviations (SD).
Unpaired t-test was used to determine the difference between two groups. Qualitative demographic and clinical data were expressed as percentages and analyzed using the chi-square test. The Kolmogorov-Smirnov test of normality was applied to all data sets. Data with nonconformance to normal distribution were analyzed using the Mann-Whitney test. A probability (p) value of $<0.05$ was considered statistically significant.

\section{Results}

\section{Clinical characteristics of the participants}

Table 1 summarizes the clinical, hormonal and metabolic data of the recruited participants. There were no significant differences $(P>0.05)$ in age, height, or BMI among the three groups among the three groups. The menstrual cycle, waist circumference, and waist-to-hip ratio of the PCOS group were significantly higher than those of the healthy control group $(\mathrm{HC})$. The waist-tohip ratio of the PCOS-IR group was higher than that of the other two groups (IR VS NIR: $0.85 \pm 0.04$ VS $0.82 \pm$ 0.03, IR VS HC:0.85 \pm 0.04 VS $0.77 \pm 0.03 P<0.05$ ). Regarding sex hormones, patients with PCOS exhibited higher levels of T, LH, LH/FSH, and lower levels of estradiol, relative to the control patients $(P<0.001)$. Nonetheless, no significant difference was observed the PCOS-IR and the PCOS-NIR group. Concerning glucose and plasma lipid levels, although fasting glucose was not different among the three groups, women with PCOS had higher fasting insulin, glycosylated hemoglobin, and homeostasis model assessment of insulin resistance (HOMA-IR). Notably, the PCOS-IR group had higher HOMA-IR values $(P<0.05)$. In contrast healthy individuals, the plasma levels of pro-inflammatory cytokines in PCOS patients, including IL-6 and TNF-a, were significantly higher. The PCOS-IR group showed higher levels of C-reactive protein, Il- 6 , TNF- $\alpha$, and free Fatty acid $($ FFA $)(P<0.05)$.

\section{Effect of PCOS on gut microbiota diversity}

These sequences comprised 297 OTUs clustered at a $97 \%$ similarity level. The dilution curve and ShannonWiener curve were used to establish whether the sequencing quantity was sufficient and estimate the species richness (Fig. 1 a). The curve seemingly flattened, indicating that the sequencing depth was sufficient to reflect the species diversity of the samples. The Venn diagram of OTU number distribution of the three groups of samples was drawn to intuitively reflect the common and unique characteristics between the groups (Fig. 1b). Alpha diversity analysis showed that, compared with the HC group, observed OTUs and Chaol index were decreased in the PCOSIR group and the PCOS-NIR group, but the differences were not statistically significant (Fig. $1 \mathrm{c}$ and 
Table 1 Clinical, biochemical and hormonal features of participants

\begin{tabular}{|c|c|c|c|}
\hline \multirow[b]{2}{*}{ Parametes } & \multirow[b]{2}{*}{$\mathrm{HC}(n=12)$} & \multicolumn{2}{|l|}{ PCOS } \\
\hline & & $\operatorname{NIR}(n=10)$ & $\mathrm{IR}(n=14)$ \\
\hline Age(years) & $28.25 \pm 1.22$ & $26.4 \pm 3.41$ & $26.71 \pm 2.43$ \\
\hline Menstrual cycle(days) & $29.67 \pm 2.02 A B$ & $82.7 \pm 26.61 B$ & $75.43 \pm 27.91 \mathrm{~A}$ \\
\hline BMI(Kg/m2) & $21.28 \pm 1.34$ & $21.32 \pm 1.22$ & $21.91 \pm 1.4$ \\
\hline Waist & $67.42 \pm 4.4 \mathrm{AB}$ & $71.8 \pm 3.29 B C$ & $77.79 \pm 5.73 \mathrm{AC}$ \\
\hline Hip & $88 \pm 5.31$ & $87.8 \pm 3.33$ & $91.54 \pm 4.43$ \\
\hline Waist to hip ratio & $0.77 \pm 0.03 \mathrm{AB}$ & $0.82 \pm 0.03 B C$ & $0.85 \pm 0.04 A C$ \\
\hline FPG (mmol/L) & $5 \pm 0.27 \mathrm{~A}$ & $5.09 \pm 0.47$ & $5.42 \pm 0.59 \mathrm{~A}$ \\
\hline FINs(uU/mL) & $6.91 \pm 1.82 \mathrm{~A}$ & $6.95 \pm 2.52 \mathrm{C}$ & $14.81 \pm 3.88 \mathrm{AC}$ \\
\hline HOMA-IR & $1.54 \pm 0.45 \mathrm{~A}$ & $1.61 \pm 0.64 \mathrm{C}$ & $3.57 \pm 1.07 A C$ \\
\hline $\mathrm{HbA1-C}$ & $5.04 \pm 0 A B$ & $5.37 \pm 0.21 B$ & $5.26 \pm 0.28 \mathrm{~A}$ \\
\hline FSH (IU/L) & $6.8 \pm 2.18$ & $5.92 \pm 2.16$ & $6.43 \pm 1.42$ \\
\hline LH(IU/L) & $6.25 \pm 2.88 \mathrm{AB}$ & $15.55 \pm 8.03 B$ & $15.37 \pm 7.31 \mathrm{~A}$ \\
\hline LH/FSH & $0.96 \pm 0.4 \mathrm{AB}$ & $2.53 \pm 0.89 \mathrm{~B}$ & $2.42 \pm 1.2 \mathrm{~A}$ \\
\hline $\mathrm{T}$ (nmol/L) & $0.75 \pm 0.21 \mathrm{AB}$ & $1.93 \pm 0.57 \mathrm{~B}$ & $1.81 \pm 0.82 \mathrm{~A}$ \\
\hline $\mathrm{P}(\mathrm{nmol} / \mathrm{l})$ & $0.54 \pm 0.16 \mathrm{~B}$ & $0.93 \pm 0.52 B$ & $0.74 \pm 0.43$ \\
\hline E2(pmol/L) & $183.51 \pm 100.9 \mathrm{~B}$ & $281.47 \pm 164.67 B$ & $194.25 \pm 55.9$ \\
\hline AMH & $4.99 \pm 3.74 \mathrm{~A}$ & $7.99 \pm 2.79$ & $9.28 \pm 5.12 \mathrm{~A}$ \\
\hline TBA (umol/l) & $4.7 \pm 2.22$ & $5.51 \pm 4.77$ & $5.26 \pm 2.4$ \\
\hline ALT (U/L) & $13.18 \pm 2.7$ & $21.02 \pm 15.13$ & $20.81 \pm 11.77$ \\
\hline AST(U/L) & $18.22 \pm 5.91$ & $22.03 \pm 6.72$ & $21.64 \pm 6.4$ \\
\hline Lithic (umol/L) & $243.43 \pm 37.04 B$ & $320.21 \pm 56.66 \mathrm{~B}$ & $283.65 \pm 105.62$ \\
\hline $\mathrm{TG}(\mathrm{mmol} / \mathrm{L})$ & $0.88 \pm 0.25 \mathrm{~A}$ & $1.14 \pm 0.84$ & $1.29 \pm 0.41 \mathrm{~A}$ \\
\hline $\mathrm{TC}(\mathrm{mmol} / \mathrm{L})$ & $4.29 \pm 1.11$ & $4.39 \pm 0.68$ & $4.77 \pm 0.88$ \\
\hline $\mathrm{HDL}(\mathrm{mmol} / \mathrm{L})$ & $1.47 \pm 0.31$ & $1.36 \pm 0.26$ & $1.33 \pm 0.23$ \\
\hline LDL(mmol/L) & $2.67 \pm 0.59$ & $2.6 \pm 0.53$ & $2.9 \pm 0.6$ \\
\hline APO-a & $93.13 \pm 71.68$ & $120.14 \pm 126.97$ & $194.49 \pm 220.43$ \\
\hline АроA/АроВ & $2.47 \pm 0.48$ & $2.31 \pm 0.56$ & $2.22 \pm 0.62$ \\
\hline $\mathrm{FFA}(\mathrm{mmol} / \mathrm{L})$ & $0.47 \pm 0.11 \mathrm{~A}$ & $0.48 \pm 0.31$ & $0.66 \pm 0.24 \mathrm{~A}$ \\
\hline hsCRP(mg/L) & $0.69 \pm 0.56 \mathrm{~A}$ & $0.8 \pm 0.75 \mathrm{C}$ & $1.63 \pm 1.27 A C$ \\
\hline IL-6(pg/ml) & $1.13 \pm 0.46 \mathrm{AB}$ & $2.12 \pm 0.26 B$ & $2.56 \pm 1.28 \mathrm{~A}$ \\
\hline TNF & $3.76 \pm 1.43 \mathrm{AB}$ & $4.86 \pm 1.07 \mathrm{~B}$ & $4.54 \pm 0.51 \mathrm{~A}$ \\
\hline
\end{tabular}

A: $P<0.05$ for HC vs. IR-PCOS group

B: $P<0.05$ for HC vs. NIR-PCOS group

C: $P<0.05$ for IR vs. NIR-PCOS group

Abbreviation: PCOS polycystic ovary syndrome; PCOS-IR PCOS with insulin resistance; PCOS-NIR PCOS withoutinsulin resistance; BMI body mass index; WHR the ratio of waist to hip; FSH follicular stimulating hormone; LHluteinizing hormone; TNF-a tumor necrosis factor-a; FPG fasting plasma glucose; FINS fasting plasma insulin;HOMAIR homeostasis model assessment of insulin resistance; PPG 3h postprandial plasma glucose; TC totalcholesterol; HDL-C high density lipoproteincholesterol; LDL-C low density lipoprotein-cholesterol; ALT alanine aminotransferase; AST aspartate aminotransferase

d). The Beta diversity analysis showed that the principal coordinate analysis (PCoA analysis) and sample clustering analysis were similar among the three groups of samples (Fig. 1e, f).

Gut microbiota composition among PCOS patients At the phylum level, Fig. 2 shows the phylum abundance distribution in the bacterial kingdom of the 36 samples, among which Firmicutes were the most abundant, followed by Proteobacteria, then Actinobacteria. The overall abundance of other bacteria was about $1 \%$. The abundance of Fusobacteria and Verrucomicrobia differed between the PCOS and the $\mathrm{HC}$ groups, but the difference was not significant $(P>0.05)$ (Fig. 2). At the family level, significant differences were observed in the abundance of 

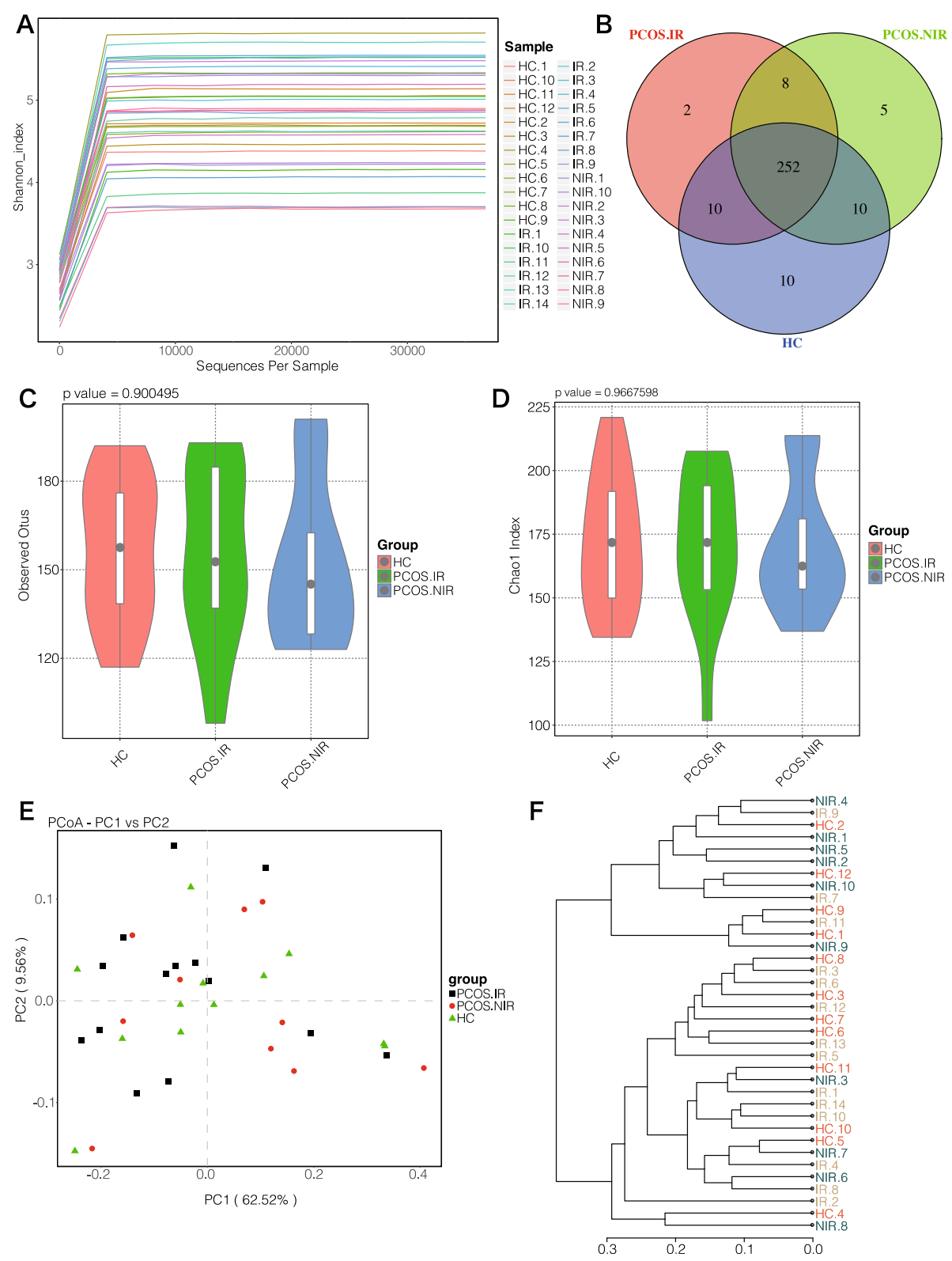

Fig. 1 Alpha and beta diversity of the gut microbial communities from participants $(n=36)$. a: Shannon-Wiener curves, showing that the amount of sequencing data is large enough to reflect the vast majority of microbial information in the samples. $\mathbf{b}$ : Venn diagram, displaying the number of common and unique OTUs, and the similarity and overlap of OTUs among groups. c: Comparison of observed OUT Numbers between the three $\operatorname{groups}(P>0.05)$. $\mathbf{d}$ : Comparisons of $C h a o 1$ indexes among the three groups $(P>0.05)$. e: Principal coordinate analysis $(P C O A)$ of fecal microbiota based on weight UniFrac metric, each dot represents the bacterial community composition of one individual stool sample, and the axis titles indicates the percentage variation explained ( 62.52 and $9.56 \%$ respectively). f: Dendrogram showing hierarchical cluster analysis based on weight UniFrac distance matrix to measure the closeness between individual samples

Lactobacillus, Enterococcaceae, Peptostreptococcaceae, and Micrococcaeae among the three groups. In the PCOS-IR group, the abundance of Peptostreptococcaceae, Enterococcaceae, and Micrococcaeae was higher than that of the other two groups. Lactobacillaceae was the highest in the PCOS-NIR group (Fig. 3). Compared with the $\mathrm{HC}$ group, PCOS patients exhibited a higher abundance of Enterococcus. The relative abundance of Rothia, Ruminococcus, Lachnospira, and Enterococcus was significantly higher in the PCOS-IR patients than in the other two groups $(P<0.05)$, whereas the abundance of the Prevotella was dramatically decreased $(P<0.05)$. Lactobacillus and Akkermansia were more abundant in the PCOS-NIR group than in the PCOS-IR and HC groups $(P<0.05)$ (Fig. 4$)$. 


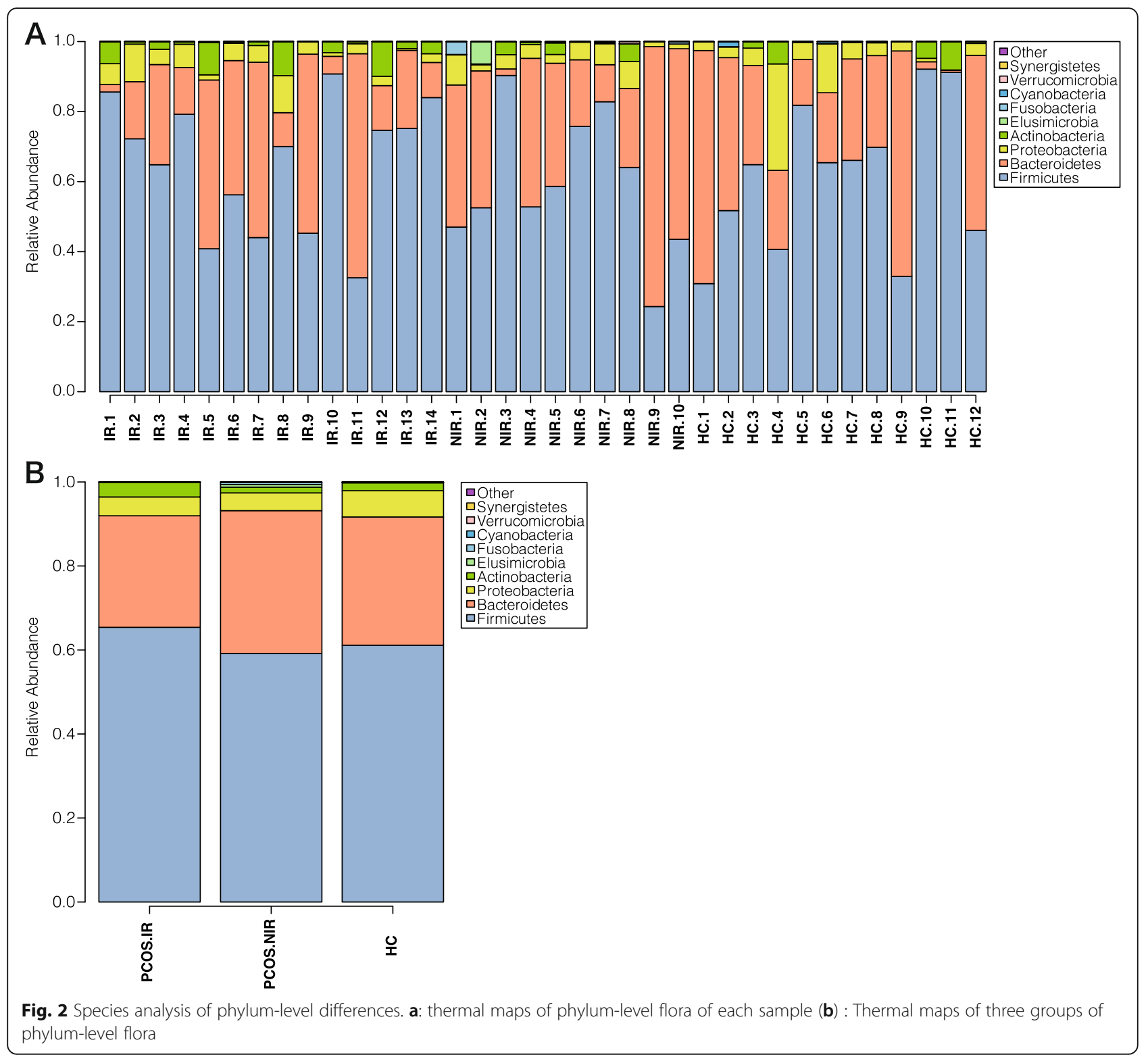

Next, to further identify species bacterial taxa with significant differences among the three groups, we used LEfSe multilevel species discrimination and LDA. Rothia and Lactobacillus were identified as the characteristic microbiota in the PCOS-IR and the PCOS-NIR groups, respectively, while the dominant bacteria in the HC group was Prevotella(LDA score > 2.0 and $P<0.05$ )(Fig. 5).

\section{Correlations between gut microbiota and metabolic parameters or sex hormones}

At the genera level, Enterococcus was positively correlated with waist circumference, hip circumference, diastolic blood pressure, and HOMA-IR index. Rothia positively correlated with waist circumference and free fatty acid (FFA) $(P<0.05)$ (Fig. 6).

\section{Discussion}

Gut microbial plays a vital role in regulating energy storage and human metabolism. As such, substantial focus has been directed to microbiota-targeted agents as novel targets for the treatment of polycystic ovary syndrome (PCOS) and related metabolic diseases. Several studies have found variations in gut microbiota composition between PCOS patients and healthy people. Besides, obese PCOS patients have been shown to exhibit more severe gut dysbiosis. However, the precise mechanism underlying the relationship between gut microbiota and the occurrence and development of 
A
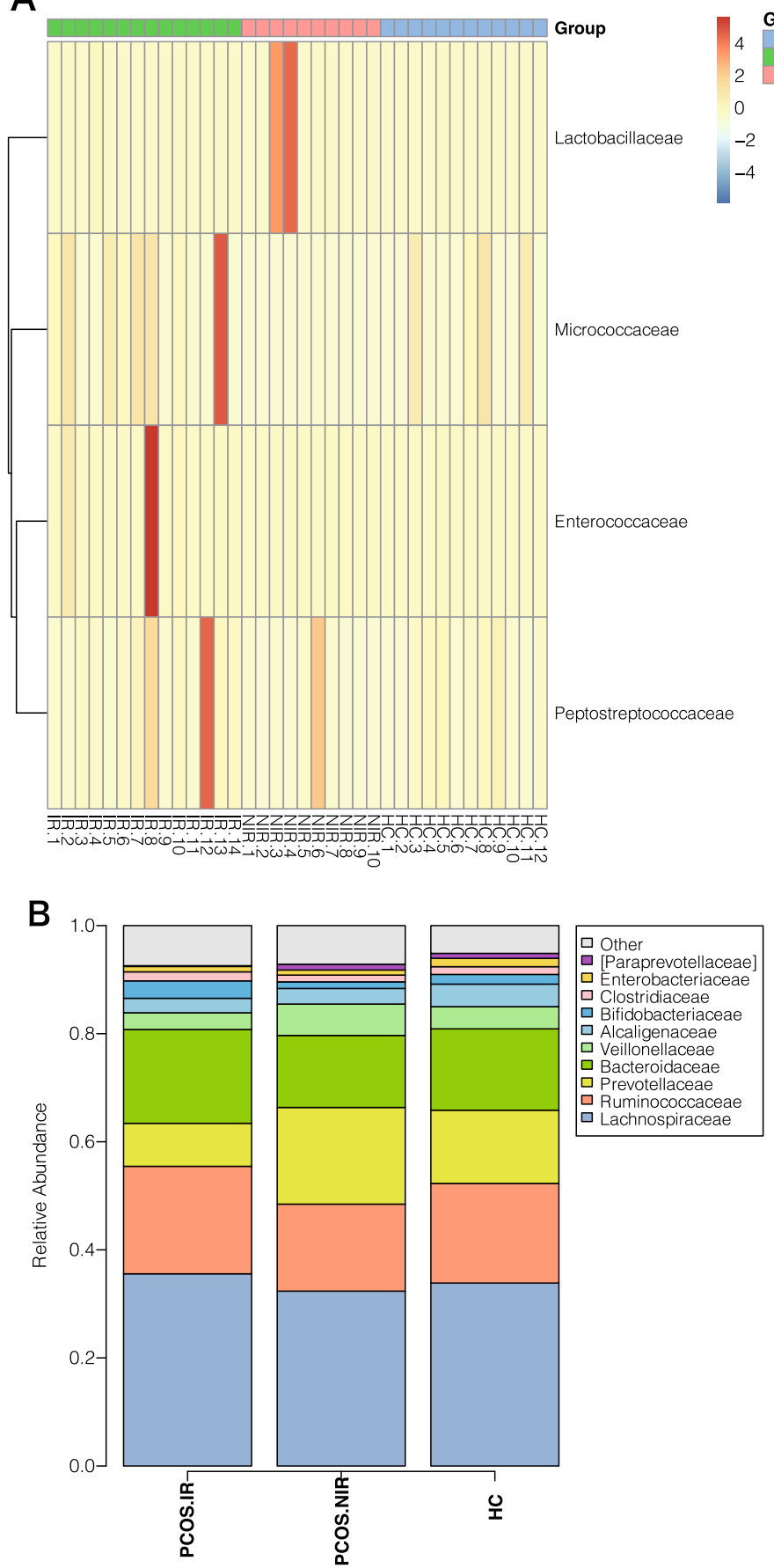

Fig. 3 Analysis of different bacterial flora at the family level (a): Bar chart of bacterial colony composition between family-level groups (b) Thermal chart of bacterial colony abundance at family level differences

PCOS remains significantly unreported. Insulin resistance (IR) is critical pathological basis of reproductive dysfunction in patients with PCOS [4-7]. Exactly $50 \%$ of patients with PCOS have IR, independent of obesity [30, 31]. However, there is limited information on the roles played by intestinal flora in the development of IR and its link with PCOS. Herein, our results revealed that gut dysbiosis was more severe in PCOS patients with insulin resistance than in the PCOS-NIR and $\mathrm{HC}$ groups. Furthermore, several taxa at the phylum level were related to the clinical characteristics of PCOS and were significantly correlated with metabolic biomarkers, including HOMA-IR, abdominal obesity, free fatty acids, and other indicators. 

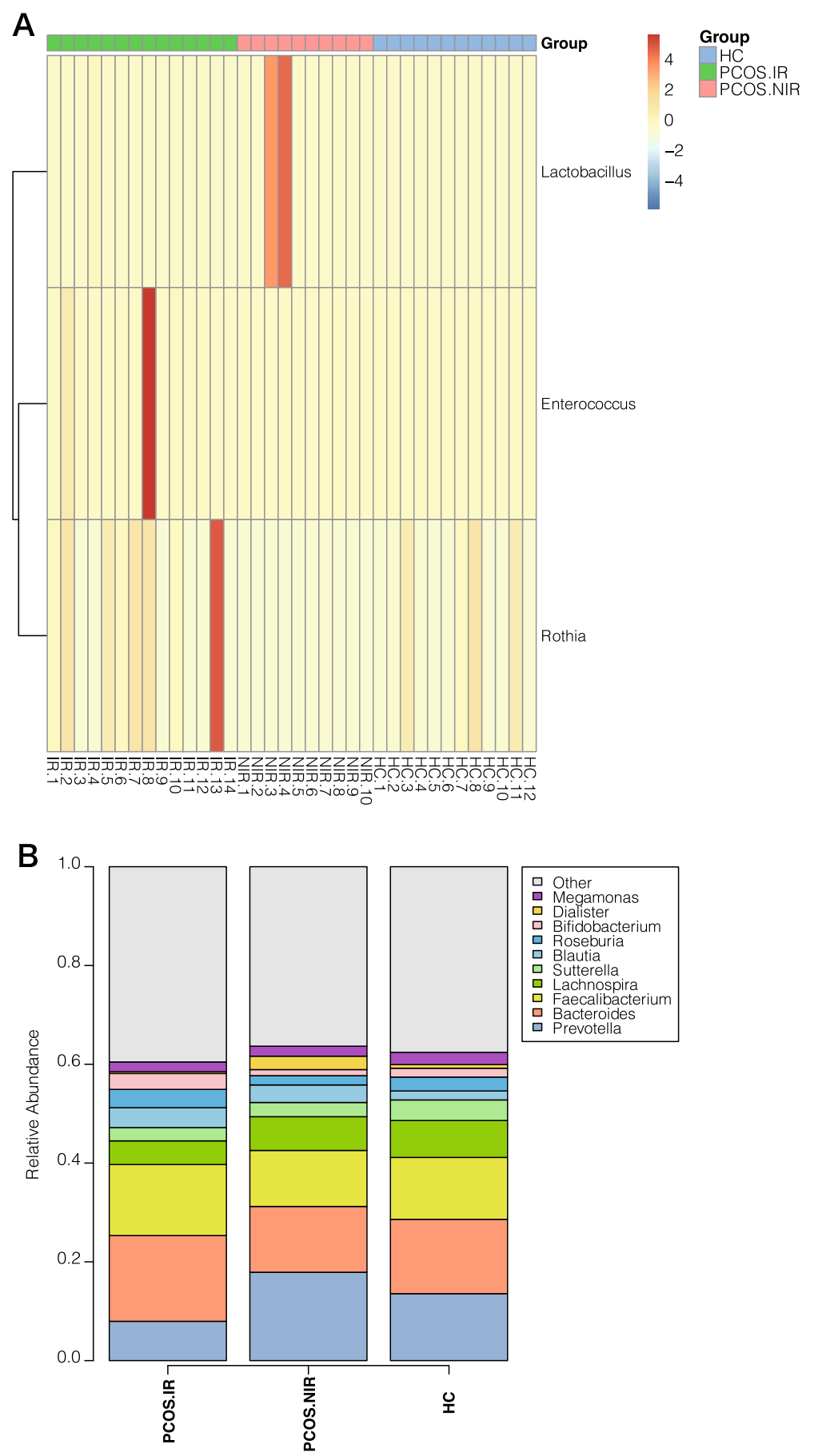

Fig. 4 Analysis of bacterial flora with difference in genera level (a): Bar chart of bacterial colony composition between genera level groups (b): Thermal chart of bacterial colony abundance with difference in genera level groups

Moreover, we showed that the composition of gut microbiota of PCOS patients with normal BMI was changed, but there was no significant difference in $\alpha$-diversity among the three groups. Noteworthy, several studies demonstrated conflicting results regarding the composition and function of the intestinal flora in PCOS patients. According to a recent meta-analysis, decreased intestinal microbiome diversity and changes in diversity are closely associated with obesity in humans [32, 33]. Previous research reported a significant decrease in gut microbiota diversity in PCOS patients or letrozole- 


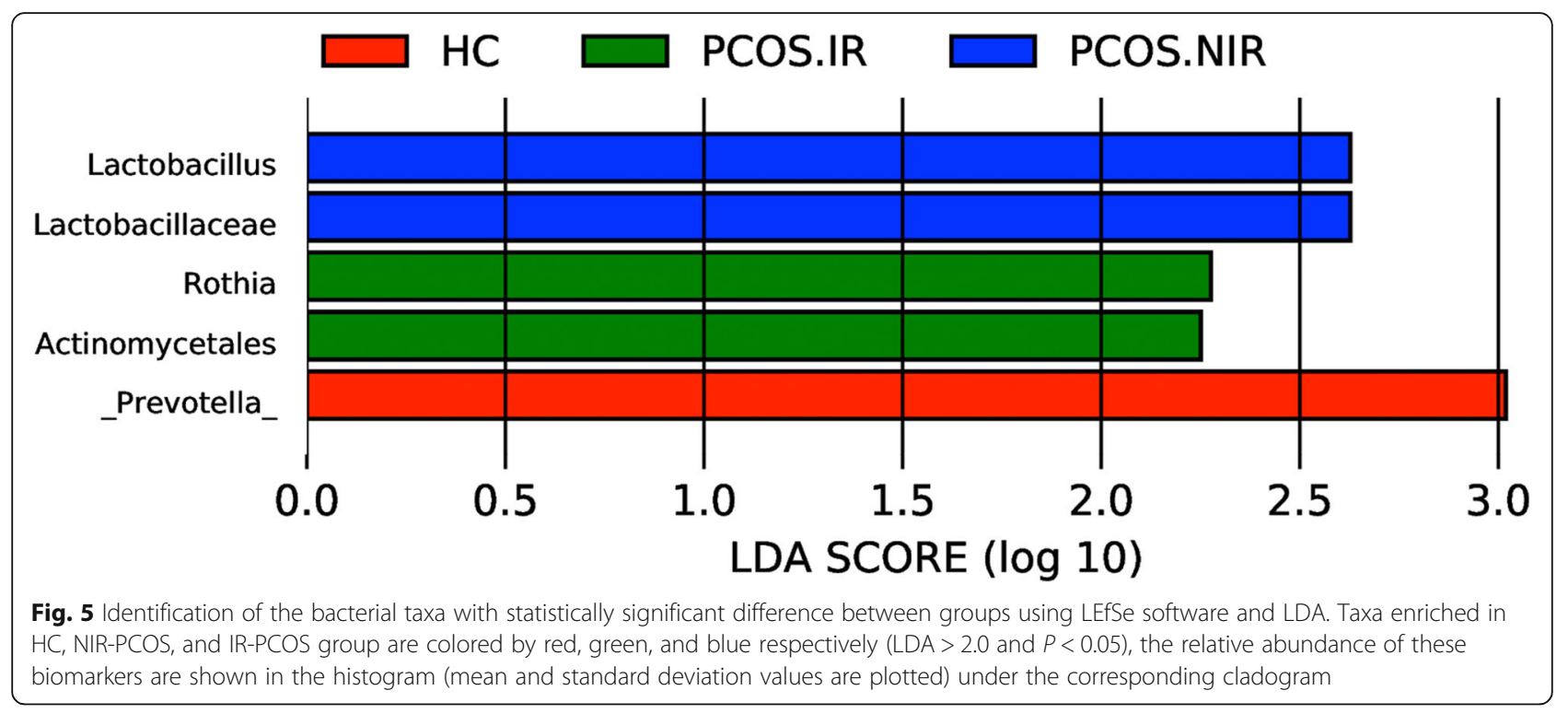

induced mouse models [19, 20, 34]. Liu et al. found that obese PCOS patients had the lowest diversity of gut microbiota [19]. Meanwhile, studies have shown that sex hormones are related to changes in gut microbiom [16, 35]. Torres et al. found that PCOS

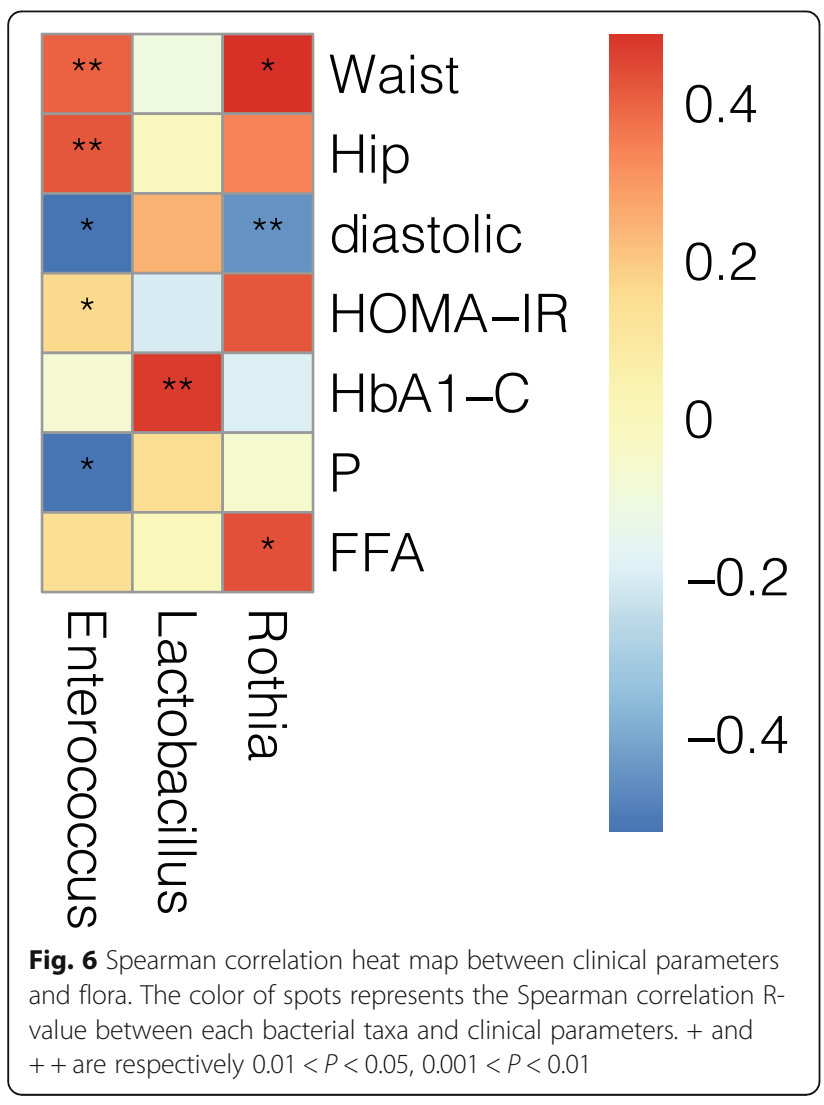

patients exhibited a lower diversity of gut microbiota than healthy controls, and that total testosterone levels were associated with reduced diversity [20]. However, Insener et al. did not find a decrease in the diversity of gut microbiota in all PCOS patients with hyperandrogenemia, whether obese or not. and diversity may be different from PCOS diagnostic criteria [21]. Similarly, in this study, $\beta$ - diversity of gut microbiota did not significantly differ among the three groups of samples based on weighted and unweighted clustering analysis. In a recent study, weighted UniFrac range-based hierarchical clustering and PCoA analysis indicated a clear distinction between the HC and the IR groups, whereas the NIR group could not be distinguished from the $\mathrm{HC}$ and IR groups [22]. Despite the absence of standard selecting sample size in microbiome studies, a study estimated that a sample size of 10 subjects per unweighted group (total sample size of 30 ) and 20 subjects per weighted group (total sample size of 60) might provide accurate statistical results for weighted analysis [36]. Besides, the $\alpha$ and $\beta$ diversity of intestinal flora may be influenced by sex, sex hormones, and obesity. In this study, the BMI of all the subjects was within the normal range, and thus the effect of obesity itself on the gut microbiota composition of the PCOS patients could not be considered. Maybe due to the small sample size, a more definite answer should be given after increasing the sample size in the future.

According to the analysis of the structural composition of gut microbiota conducted in this study, the three groups were mainly composed of Bacteroidetes and Firmicutes at the phylum level. At the genus level, the 
relative abundance of Enterococcus in the PCOS group increased significantly $(\mathrm{P}<0.05)$ and was highest in the IR group. Enterococcus is a common gram-positive bacteria and can be divided into five categories according to phylogenetic similarity. Among them, Enterococcus faecalis and Enterococcus faecium are the major pathogenic bacteria in humans [37, 38]. Although the causal role of genus Enterococcus in the occurrence and development of metabolic diseases has not been fully revealed, previous studies have found that Enterococcus is more abundant in the gut microbiota of obese children and adolescents [39], as well as in mice under a high-fat/ high-sugar "Western" diet [40]. A recent study found that Enterococcus can regulate the level of incretin hormone glucagon-like peptide-1 (GLP-1). GeIE secreted by E. fasecalis can degrade GLP-1 (GLP-1), causing abnormal insulin secretion[41]. At the same time, GeIE can degrade intestinal gastric inhibitory peptides (just like leptin), and thereby interfere with the metabolism of the host. Similarly, in our study, Enterococcus was the most abundant genera in the PCOS-IR group, and its abundance was positively correlated with insulin resistance index. GLP-1 plays a role in regulating glucose homeostasis and reducing appetite in the body. Considering the vital role of GLP-1 in the development of type 2 diabetes and other metabolic diseases, we speculated that Enterococcus could influence the occurrence and development of PCOS by regulating the GLP-1 signaling pathway, specifically in patients with IR. Studies have shown that after oral glucose tolerance tests, GLP-1 activity in lean PCOS patients is usually lower than in healthy women [42]. The use of GLP-1 receptor agonists in the treatment of PCOS patients enhances symptoms and reduces metabolic complications by reducing weight and insulin resistance [43, 44]. Insulin secretion and gastric emptying are affected by the intestinal flora environment and intestinal flora imbalance. Estelle et al. proved the important role of intestinal flora in controlling GLP-1induced insulin secretion and gastric emptying in mice [45]. Therefore, the role of Enterococcus in the regulation of GLP-1 level in PCOS patients should be explored further.

The critical bacterial genus in the intestinal tract of patients with PCOS was identified using LEfSe multilevel species discrimination and LDA. Consequently, Rothia played vital roles in the PCOS-IR group, whereas Prevotella was the dominant bacterial group in the $\mathrm{HC}$ group. Rats fed a high-fat diet exhibited significantly increased fat, reduced insulin sensitivity, increased abundance of esophageal Rothia, and Rothia were associated with fasting blood glucose and insulin. These observations were linked the expression of inflammatory genes and fatty acid transport and metabolism in the esophagus
[46]. Changes in Rothia flora in the adolescent oral cavity were associated with obesity [47]. Women with gestational diabetes have also shown an increase in Rothia abundance, relative to women with normal blood sugar [48]. In this study, Rothia had significant advantages in the PCOS-IR group, and its abundance was positively correlated with waist circumference and free fatty acid (FFA). Previous studies have shown that non-obese PCOS patients with insulin resistance have a more remarkable centra distribution of fat [37]. In our research, PCOS patients with normal BMI exhibited abdominal fat accumulation. Besides, Rothia abundance in the PCOS-IR group was related to abdominal obesity. Intestinal flora may participate visceral fat metabolism, release excess too much free fatty acids, increase lipotoxicity, and reducing insulin sensitivity. Prevotella is a bacterium that produces short-chain fatty acids (SCFA), regulates the uptake of nutrients and hormone levels in the gut. Also, it participates in energy metabolism, and its decreased abundance is significantly associated with increased testosterone and pro-inflammatory cytokines [22]. In this study, the HC group had the highest abundance of Prevotella, maintaining the balance of intestinal flora, while the NIR and IR groups had a reduced abundance of Prevotella. However, there was no correlation between the decreased abundance of the Prevotella and the sex hormones and biochemical indicators, potentially due to a small sample size used. The relationship between PCOS and intestinal flora is complex and could be related to genetic, lifestyle, and environmental factors [49, 50]. We excluded the influence of smoking, taking antibiotics and probiotics on intestinal flora in the included subjects. However, distinct backgrounds (including region, race, lifestyle, and diet habit) of the subjects could be responsible for the differences in the PCOS-related intestinal flora [51].

\section{Conclusion}

In conclusion, we analyzed the composition of the intestinal microbiota in PCOS patients with normal BMI combined with insulin resistance and its relationship with clinical characteristics. Notably, no significant difference was found in intestinal microbial diversity among the three groups of patients. Nonetheless, the abundance of Enterococcus and Rothia significantly increased in the PCOS-IR group and correlated with insulin resistance, suggesting that the bacterium potentially plays a key role in the pathogenesis of PCOS by regulating the glucagon-like peptidin-1 (GLP-1) level and other mechanisms. The altered intestinal flora might affect the intestinal environment of the host by enriching different 
metabolic functions and promoting the development of PCOS insulin resistance and disease in women. This experimental cross-sectional study involved participants from different regions, RACES, and eating habits, which might have brought heterogeneity. Considering the variations in human intestinal microbiota, many clinical population and animal experiments are essential to validate our findings. Also, further studies are imperative to elucidate the precise function of various flora and the mechanism underlying their roles in the pathogenesis of multiple diseases. Therefore our next task will involve performing a metabolomic analysis to explore further the relationship between insulin resistance and intestinal flora among PCOS patients. These findings will provide a theoretical basis for drug design and subsequent clinical treatment of PCOS.

\section{Abbreviations \\ PCOS: Polycystic ovary syndrome; IR: Insulin resistance; BCAA: Branched chain amino acid; SCFA: Short chain fatty acid; HA: Hyperandrogenism; \\ P: Progesterone; PRL: Prolactin; T: Testosterone; LH: Luteinizing hormone; FSH: Follicle-stimulating hormone; E2: Oestradiol; SHBG: Sex binding globulin; AMH: Anti-Mullerian hormone; TG: Triglyceride; TC: Total cholesterol; LDL: Low-density lipoprotein; HDL: High-density lipoprotein; CRP: C-reactive protein; IL-6: Interleukin-6; TNF-a: Tumor necrosis factor; OUT: Operational Taxonomic Units; LDA: Linear discriminant analysis; FFA: Free fatty acid; GLP- 1: Glucagon-like peptide-1}

\section{Acknowledgements}

We are grateful to the women who participated in the survey.

\section{Authors' contributions}

All authors contributed to the concepts of the manuscript; Fangfang He: Data collection, analysis, and management, manuscript writing. Yumei Li: Project development, manuscript writing and editing. All authors read and approved the final manuscript.

\section{Funding}

Not applicable.

\section{Availability of data and materials}

The datasets are available from the corresponding author on reasonable request.

\section{Declarations}

\section{Ethics approval and consent to participate}

This study was approved by the Ethics Committee of the Department of Assisted Reproduction(Xiangya Hospital,Central South University,)as well as the China Registered Clinical Trial Ethics Review Committee (Ethical Review No.: CHiECRT1900028223).

\section{Consent for publication}

This manuscript was an original research that has not been published previously, and not under consideration for publication elsewhere, in whole or in part.

\section{Competing interests}

The authors have no conflicts of interest to disclose.
Received: 29 November 2020 Accepted: 17 March 2021

Published online: 27 March 2021

\section{References}

1. Sahmay S, Aydogan Mathyk B, Sofiyeva N, Atakul N, Azemi A, Erel T. Serum $\mathrm{AMH}$ levels and insulin resistance in women with PCOS. Eur J Obstet Gynecol Reprod Biol. 2018;224:159-64.

2. Norman RJ, Dewailly D, Legro RS, Hickey TE. Polycystic ovary syndrome. The Lancet. 2007;370(9588):685-97.

3. Rosenfield RL, Ehrmann DA. The Pathogenesis of Polycystic Ovary Syndrome (PCOS): The Hypothesis of PCOS as Functional Ovarian Hyperandrogenism Revisited. Endocr Rev. 2016;37(5):467-520.

4. Macut D, Bjekic-Macut J, Rahelic D, Doknic M. Insulin and the polycystic ovary syndrome. Diabetes Res Clin Pract. 2017;130:163-70.

5. Diamanti-Kandarakis $\mathrm{E}$, Dunaif $\mathrm{A}$. Insulin resistance and the polycystic ovary syndrome revisited: an update on mechanisms and implications. Endocr Rev. 2012;33(6):981-1030.

6. Legro RS, Arslanian SA, Ehrmann DA, Hoeger KM, Murad MH, Pasquali R, et al. Diagnosis and treatment of polycystic ovary syndrome: an Endocrine Society clinical practice guideline. J Clin Endocrinol Metab. 2013;98(12): 4565-92.

7. Rotterdam EA-SPcwg. Revised 2003 consensus on diagnostic criteria and long-term health risks related to polycystic ovary syndrome (PCOS). Hum Reprod. 2004;19(1):41-7.

8. Garg D, Tal R. Inositol Treatment and ART Outcomes in Women with PCOS. Int J Endocrinol. 2016;2016:1979654.

9. Goodman NF, Cobin RH, Futterweit W, Glueck JS, Legro RS, Carmina E, et al. American Association of Clinical Endocrinologists, American College of Endocrinology, and Androgen Excess and Pcos Society Disease State Clinical Review: Guide to the Best Practices in the Evaluation and Treatment of Polycystic Ovary Syndrome - Part 2. Endocr Pract. 2015;21(12):1415-26.

10. Coyte KZ, Schluter J, Foster KR. The ecology of the microbiome: Networks, competition, and stability. Science. 2015;350(6261):663-6.

11. Hartstra AV, Bouter KE, Backhed F, Nieuwdorp M. Insights into the role of the microbiome in obesity and type 2 diabetes. Diabetes Care. 2015;38(1): 159-65.

12. Boulange $\mathrm{CL}$, Neves $\mathrm{AL}$, Chilloux J, Nicholson JK, Dumas ME. Impact of the gut microbiota on inflammation, obesity, and metabolic disease. Genome Med. 2016;8(1):42.

13. Scheithauer TP, Dallinga-Thie GM, de Vos WM, Nieuwdorp M, van Raalte DH Causality of small and large intestinal microbiota in weight regulation and insulin resistance. Mol Metab. 2016;5(9):759-70.

14. Jiao N, Baker SS, Nugent CA, Tsompana M, Cai L, Wang Y, et al. Gut microbiome may contribute to insulin resistance and systemic inflammation in obese rodents: a meta-analysis. Physiol Genomics. 2018;50(4):244-54.

15. Tremellen K, Pearce K. Dysbiosis of Gut Microbiota (DOGMA)--a novel theory for the development of Polycystic Ovarian Syndrome. Med Hypotheses. 2012;79(1):104-12

16. Thackray VG. Sex, Microbes, and Polycystic Ovary Syndrome. Trends Endocrinol Metab. 2019;30(1):54-65

17. Zhao X, Jiang $Y, X i H$, Chen L, Feng X. Exploration of the Relationship Between Gut Microbiota and Polycystic Ovary Syndrome (PCOS): a Review. Geburtshilfe Frauenheilkd. 2020;80(2):161-71.

18. Lindheim L, Bashir M, Munzker J, Trummer C, Zachhuber V, Leber B, et al. Alterations in Gut Microbiome Composition and Barrier Function Are Associated with Reproductive and Metabolic Defects in Women with Polycystic Ovary Syndrome (PCOS): A Pilot Study. PLoS One. 2017;12(1): e0168390.

19. Liu R, Zhang C, Shi Y, Zhang F, Li L, Wang X, et al. Dysbiosis of Gut Microbiota Associated with Clinical Parameters in Polycystic Ovary Syndrome. Front Microbiol. 2017;8:324.

20. Torres PJ, Siakowska M, Banaszewska B, Pawelczyk L, Duleba AJ, Kelley ST, et al. Gut Microbial Diversity in Women With Polycystic Ovary Syndrome Correlates With Hyperandrogenism. J Clin Endocrinol Metab. 2018;103(4): 1502-11.

21. Insenser M, Murri $M$, Del Campo R, Martinez-Garcia MA, Fernandez-Duran E, Escobar-Morreale HF. Gut Microbiota and the Polycystic Ovary Syndrome: Influence of Sex, Sex Hormones, and Obesity. J Clin Endocrinol Metab. 2018; 103(7):2552-62. 
22. Zeng B, Lai Z, Sun L, Zhang Z, Yang J, Li Z, et al. Structural and functional profiles of the gut microbial community in polycystic ovary syndrome with insulin resistance (IR-PCOS): a pilot study. Res Microbiol. 2019;170(1):43-52.

23. He W, Li Q, Yang M, Jiao J, Ma X, Zhou Y, et al. Lower BMl cutoffs to define overweight and obesity in China. Obesity (Silver Spring). 2015;23(3):684-91.

24. Oakkar EE, Stevens J, Truesdale KP, Cai J. BMI and all-cause mortality among Chinese and Caucasians: the People's Republic of China and the Atherosclerosis Risk in Communities Studies. Asia Pac J Clin Nutr. 2015;24(3): 472-9.

25. YingYing S. Clinical research on insulin releasing curve rating method in evaluation of insulin resistance in patients with PCOS. China Academic Jounal Electronic Publishing. 2015;30(20).

26. Caporaso JG, Kuczynski J, Stombaugh J, Bittinger K, Bushman FD, Costello EK, et al. QIIME allows analysis of high-throughput community sequencing data. Nat Methods. 2010;7(5):335-6.

27. Edgar RC, Haas BJ, Clemente JC, Quince C, Knight R. UCHIME improves sensitivity and speed of chimera detection. Bioinformatics. 2011;27(16): 2194-200

28. DeSantis TZ, Hugenholtz P, Larsen N, Rojas M, Brodie EL, Keller K, et al. Greengenes, a chimera-checked 16S rRNA gene database and workbench compatible with ARB. Appl Environ Microbiol. 2006;72(7):5069-72.

29. Feng Q, Liang S, Jia H, Stadlmayr A, Tang L, Lan Z, et al. Gut microbiome development along the colorectal adenoma-carcinoma sequence. Nature communications. 2015;6:6528.

30. Widecka J, Ozegowska K, Banaszewska B, Kazienko A, Safranow K, BraneckaWozniak D, et al. Is copeptin a new potential biomarker of insulin resistance in polycystic ovary syndrome? Ginekol Pol. 2019;90(3):115-21.

31. Barber TM. Divergences in insulin resistance between the different phenotypes of the polycystic ovary syndrome. Expert Rev Endocrinol. 2013; 8(5):427-9.

32. Sze MA, Schloss PD. Looking for a Signal in the Noise: Revisiting Obesity and the Microbiome. MBio. 2016;7(4)

33. Walters WA, Xu Z, Knight R. Meta-analyses of human gut microbes associated with obesity and IBD. FEBS Lett. 2014;588(22):4223-33.

34. Kelley ST, Skarra DV, Rivera AJ, Thackray VG. The Gut Microbiome Is Altered in a Letrozole-Induced Mouse Model of Polycystic Ovary Syndrome. PLoS One. 2016;11(1):e0146509.

35. Sherman SB, Sarsour N, Salehi M, Schroering A, Mell B, Joe B, et al. Prenatal androgen exposure causes hypertension and gut microbiota dysbiosis. Gut Microbes. 2018:1-22.

36. Kelly BJ, Gross R, Bittinger K, Sherrill-Mix S, Lewis JD, Collman RG, et al. Power and sample-size estimation for microbiome studies using pairwise distances and PERMANOVA. Bioinformatics. 2015;31(15):2461-8.

37. Byappanahalli MN, Nevers MB, Korajkic A, Staley ZR, Harwood VJ. Enterococci in the Environment. Microbiol Mol Biol Rev. 2012;76(4):685-706.

38. Ruzickova M, Vitezova M, Kushkevych I. The Characterization of Enterococcus Genus: Resistance Mechanisms and Inflammatory Bowel Disease. Open Med (Wars). 2020;15:211-24.

39. Hou YP, He QQ, Ouyang HM, Peng HS, Wang Q, Li J, et al. Human Gut Microbiota Associated with Obesity in Chinese Children and Adolescents. Biomed Res Int. 2017;2017:7585989.

40. Turnbaugh PJ, Ridaura VK, Faith JJ, Rey FE, Knight R, Gordon Jl. The effect of diet on the human gut microbiome: a metagenomic analysis in humanized gnotobiotic mice. Sci Transl Med. 2009;1(6):6ra14.

41. LeValley SL, Tomaro-Duchesneau C, Britton RA. Degradation of the Incretin Hormone Glucagon-Like Peptide-1 (GLP-1) by Enterococcus faecalis Metalloprotease GelE. mSphere. 2020;5(1).

42. Vrbikova J, Hill M, Bendlova B, Grimmichova T, Dvorakova K, Vondra K, et al. Incretin levels in polycystic ovary syndrome. European journal of endocrinology. 2008;159(2):121-7.

43. Lamos EM, Malek R, Davis SN. GLP-1 receptor agonists in the treatment of polycystic ovary syndrome. Expert Rev Clin Pharmacol. 2017;10(4):401-8.

44. Niafar M, Pourafkari L, Porhomayon J, Nader N. A systematic review of GLP-1 agonists on the metabolic syndrome in women with polycystic ovaries. Arch Gynecol Obstet. 2016;293(3):509-15.

45. Grasset E, Puel A, Charpentier J, Collet X, Christensen JE, Tercé F, et al. A Specific Gut Microbiota Dysbiosis of Type 2 Diabetic Mice Induces GLP-1 Resistance through an Enteric NO-Dependent and Gut-Brain Axis Mechanism. Cell Metab. 2017;26(1):278.
46. Kaakoush NO, Lecomte V, Maloney CA, Morris MJ. Cross-talk among metabolic parameters, esophageal microbiota, and host gene expression following chronic exposure to an obesogenic diet. Sci Rep. 2017;7:45753.

47. de Andrade PAM, Giovani PA, Araujo DS, de Souza AJ, Pedroni-Pereira A, Kantovitz KR, et al. Shifts in the bacterial community of saliva give insights on the relationship between obesity and oral microbiota in adolescents. Arch Microbiol. 2020.

48. Crusell MKW, Hansen TH, Nielsen T, Allin KH, Rühlemann MC, Damm P, et al. Gestational diabetes is associated with change in the gut microbiota composition in third trimester of pregnancy and postpartum. Microbiome. 2018;6(1).

49. Vink JM, Sadrzadeh S, Lambalk CB, Boomsma DI. Heritability of polycystic ovary syndrome in a Dutch twin-family study. J Clin Endocrinol Metab. 2006;91(6):2100-4.

50. Domecq JP, Prutsky G, Mullan RJ, Hazem A, Sundaresh V, Elamin MB, et al. Lifestyle Modification Programs in Polycystic Ovary Syndrome: Systematic Review and Meta-Analysis. The Journal of Clinical Endocrinology Metabolism. 2013;98(12):4655-63.

51. Knight R, Vrbanac A, Taylor BC, Aksenov A, Callewaert C, Debelius J, et al. Best practices for analysing microbiomes. Nat Rev Microbiol. 2018;16(7): $410-22$.

\section{Publisher's Note}

Springer Nature remains neutral with regard to jurisdictional claims in published maps and institutional affiliations.
Ready to submit your research? Choose BMC and benefit from:

- fast, convenient online submission

- thorough peer review by experienced researchers in your field

- rapid publication on acceptance

- support for research data, including large and complex data types

- gold Open Access which fosters wider collaboration and increased citations

- maximum visibility for your research: over $100 \mathrm{M}$ website views per year

At $\mathrm{BMC}$, research is always in progress.

Learn more biomedcentral.com/submissions 